\title{
Space, Time, and the Creation of Universe
}

\author{
Zhi Gang Sha \\ Institute of Soul Healing and Enlightenment
}

\author{
Rulin Xiu \\ Institute of Soul Healing and Enlightenment
}

\begin{abstract}
The understanding about the creation of our universe is explored in many philosophies, natural sciences, religions, ideologies, traditions, and disciplines. Current natural science cannot answer this question at the fundamental level. In this work, we combine the ancient Chinese Tao wisdom about the creation with quantum physics. We propose that everything comes from the emptiness. Our universe is manifested from the emptiness through two pairs of duality measurements: space and time duality pair and inclusion and exclusion duality pair. From this understanding, we are able to derive one mathematic formula describing our universe. It also yields a new metaphysical approach to derive and interpret string theory as well as produce more testable predictions from string theory. This work gives a new way to understand and mathematically describe how our universe is created and evolved. It provides another way to comprehend the meaning and function of space and time. It indicates that our universe is manifested from the emptiness through human actions. Space and time is a pair of duality action and codes that help manifest our universe. It provides answer to an important philosophical question about whether and why we can understand and mathematically describe our universe.
\end{abstract}

Keywords: space, time, creation of universe, string theory, Tao wisdom, quantum physics, measurement theory, duality, manifestation, grand unification theory

Understanding the secret about creation is one of the highest wisdom, knowledge, and enlightenment a human being can dream about. This wisdom and knowledge will not only empower us to become a powerful manifest or, more importantly, it is essential for liberating humanity from illusions, sufferings, limitations, lacking, ignorance, and bondage. This wisdom is the doorway to a higher level of consciousness and enlightenment.

More than 300 years ago, Issac Newton, together with other pioneers, enlightened humanity with the profound truth that planets, stars, mother earth, humane, and all things follow the same physics law. With the language of our mind, the mathematics, we can understand and grasp the natural laws that govern everyone and everything. This sets up the foundation for what we now call scientific study, which is to use mathematic formula to express repeatable experiment to seek deeper understanding of nature. The founding of scientific study has deep and powerful impact on humanity. The whole history of humanity has changed because of it. Since then, physics and natural science has been playing more and more important role in our lives, societies, and world. Humanity's power to impact nature started to grow exponentially.

Zhi Gang Sha, Dr., Institute of Soul Healing and Enlightenment, Canada; main research fields: Tao Wisdom and Practice, Tao Healing, and Tao Calligraphy, Soul Mind Body Science System, and Tao Science.

Rulin Xiu, Ph.D., Institute of Soul Healing and Enlightenment and Hawaii Theoretical Physics Research Center, USA; main research field: Theoretical Physics, String Theory, Grand Unification Theory, Soul Mind Body Science System, and Tao Science.

Acknowledgements: We want to thank Dr. Ervin Laszlo and many others for their help, support, and encouragement with this project. I want to thank Marsha Vaulutis, Alan Chuck, and Christine Falcon for editing this paper. 
Quantum physics is the most fundamental physics theory so far. It has been shown that classical physics can be derived from quantum physics mathematically as an approximation. Quantum physics yields the most accurate predictions about nature. It has expanded our knowledge about nature tremendously, in areas such as chemistry, material science, atomic physics, nuclear physics, and particle physics, as well as astrophysics, cosmology, and more. It has also led to an immense number of new inventions and technologies.

Quantum physics studies what everything is made of and how it behaves at the microscopic level. Studies in quantum physics indicate that everything is made of various vibrations, also called waves. Vibration or wave is the same as periodic oscillation. Because quantum vibrations are not limited by space and time, everything is basically a vibrational field consisting of different vibrations. It is described by wave function mathematically. Wave function is the mathematical formula that expresses the types and quantity of vibrations or waves inside a system.

Quantum physics drastically challenges the foundation of natural science in three ways: predictability, objectivity, and locality of natural phenomena. Understanding quantum physics metaphysically is still an active pursuit in physics and philosophy. Many scientists question whether quantum physics is a complete or correct theory about reality. Many interpretations have been proposed to make sense of quantum physics.

Astrophysics and cosmology attempt to explore our universe with insight, mathematic formula and experimental data. Astrophysics is a branch of physics that studies what is made of the heavenly bodies, such as sun, stars, galaxies, extrasolar planets, interstellar medium, and the cosmic microwave background. Cosmology studies the origin, evolution, large-scale structures, dynamics, and ultimate fate of the universe, as well as the scientific laws that govern the universe.

Much observation data indicate that our universe starts from a big bang, followed almost instantaneously by cosmic inflation, an expansion of space from which the universe is thought to have emerged 13.799 billion years ago. The existence of dark energy is proposed to describe the unknown energy source for the observed accelerated expansion of our universe. Dark matter is suggested to account for the unknown matter necessary for the appearance of the large-scale structure detected in our universe.

The grand unification theory, also called the theory of everything, is the attempt to use one mathematic formula to explain all the fundamental forces and matters discovered so far. String theory is a promising candidate for the grand unification theory. String theory studies the quantum dynamics of a string. The vibration of string creates particles and forces. String theory has the potential to predict the dimension of space-time, the particle and interaction spectrum, to unify all the forces and fundamental particles, and more. However, with all these great potentials and promises, string theory has not yet made many testable predictions. Something is still missing if string theory will ever become the grand unification theory.

Even with all its achievement, current physics, astrophysics, cosmology, string theory, as well as all the candidates of grand unification theory, cannot answer the question about where our universe comes from and how it is created at the fundamental level. In this paper, we explore to apply Tao wisdom about the creation to the theoretical physics. We will mathematically formulate Tao wisdom and derive the one formula about our universe. We will first give a summary of Tao wisdom about creation. Then we will explore the meaning and function of space-time at a fundamental level. We propose that at the deeper level, space-time is one of the two yin-yang pairs that manifest our universe from the emptiness. In the end, we will discuss the significance of this finding for philosophy, physics, and development of humanity in general. 


\section{Ancient Tao Wisdom}

Tao is an ancient Chinese natural philosophy, tradition, ethic, spiritual practice, and wisdom with a written history of about 5000 years. It has greatly influenced Chinese culture in every aspect. It starts to impact more and more people worldwide.

According to Tao wisdom, Tao is the emptiness. It is the origin and creator of everyone, everything, and our whole universe. Everything comes in existence through yin yang interaction. Yin yang is the basic universal code and driving force behind all creation and change in the universe.

Yin yang is one of the most ancient Chinese wisdom and concept. This wisdom and concept is the origin and foundation for all Chinese wisdom, culture, logic, ideology, and philosophy. Yin and yang represent two opposite elements existing in everyone and everything. Usually, yin represents the part that is passive, dark, contracting, feminine, down. Yang represents the part that is active, light, expanding, masculine, and up.

The wisdom of yin yang tells us that everyone and everything consist of both yin and yang elements. The relation between yin and yang includes four aspects:

(1) Yin and yang are opposite and relative

(2) Yin and yang are co-created

(3) Yin and yang are inseparable and co-dependent

(4) The interaction of yin and yang creates everyone and everything. It is the cause of all changes.

In ancient Chinese book, Yellow Emperor's Cannon, it is said: "Yin yang is the universal principle. It is the fundamental law followed by everyone and everything. It is the origin of all changes. It is the cause of birth and death. It is the reason of all creation."

According to Tao wisdom, Tao is the emptiness. Tao creates everyone and everything through the Tao normal creation process: "Tao Sheng Yi. Yi Sheng Er. Er Sheng San. San Sheng Wan Wu."

Here Sheng means create. Yi means one. Er means two. San means three. Wang wu means all things. The literal translation of the above phrase is:

Tao creates One. One creates Two. Two creates Three. Three creates All Things.

This ancient text tells us that Tao creates a oneness state. This oneness state can split into a yin yang pair. Yin, Yang, and Tao, these three elements, create all things.

\section{The Quantum Physics about Tao}

What is Tao according to physics? Can science help us understand Tao?

In physics, emptiness is sometimes called vacuum. In classical physics, emptiness is nothingness. There is nothing in the emptiness. In quantum physics, the emptiness is not nothingness any longer. Vibrations can pop out of as well as go back to the emptiness. This phenomenon is the so called the vacuum fluctuation. Since there is no blockage in the emptiness, all sorts of vibrations can show up in the emptiness through the quantum fluctuation.

In fact, when quantum physicists attempt to calculate the information, energy, and matter in the emptiness, they encounter the infinity. Infinity is something so huge or so small it cannot be counted. It is bigger than the biggest. It is smaller than the smallest. Within emptiness, there are countless information, energy, and matter.

Infinity is one of the most challenging problems physicists have ever run into. In fact, encountering infinity is quite a scaring experience. At the beginning, physicists choose to ignore the infinity associated with 
the emptiness. After all, nothing in this world is emptiness. However, they cannot shun away from the infinity and the emptiness very long. Soon they find that, in quantum physics, matter interacts with the emptiness. This interaction can also create infinity.

To avoid the infinity problems associated with the emptiness, physicists came up with renormalization procedure to calculate how the emptiness affects the measurable quantities, such as charge, mass, energy, and other observable quantities. Renormalization theory reveals one important fact: Natural phenomena may appear different when observed at different space and time scale.

Let's summarize what quantum physics tells us about Tao, the emptiness:

(1) Emptiness contains boundless information, energy, and matter.

(2) Emptiness is within everything.

(3) Emptiness interacts with everything.

\section{The Quantum Physics about Creation}

Quantum phenomena challenge one of the corner stones of natural science: objectivity. It is generally accepted in natural science that natural phenomena are objective. Their existence does not depend on the action of the observer. In quantum physics, however, phenomena are subjective and depend on the action of observers.

In quantum physics, the world we observed is determined by our action of the observer. Quantum measurement is the action of observation. The measurement process is a determining factor for what is being observed. It is in fact part of the process that creates the quantum phenomena. The world we observed is manifested by our measurement in quantum physics.

This is a revolutionary revelation. The implication of this revelation has not been paid enough attention by most physicists and scientists. In the following we will show, the combination of the full grasp of this revelation with the Tao wisdom can help us understand the creation of our universe and come up with the grand unification theory.

\section{Meaning and Function of Space and Time}

How does the yin yang interaction manifest the universe from the emptiness? To answer this question, we first need to find out the yin yang pairs that create our universe. To do this, we need to understand what space and time is, as the creation of our universe relates to space and time.

Space and time are two mysteries that have been explored by all people in all cultures, traditions, ideologies, philosophies, and sciences in history. Space and time have many layers of meaning and applications. For instance, some people think time is life. Some people consider time and space is money.

In the 17th century, understanding about space and time emerged as a central issue in founding science. Gottfried Leibniz and Issac Newton led two opposing theories about space and time. Gottfried Leibniz, a German philosopher-mathematician, believes that space and time does not exist. They are no more than the collection of and an idealized abstraction from the relations between objects and events.

Issac Newton, the founder of classical mechanics, views space and time as absolute in the sense that it exists permanently and independently of whether there was any matter or observer.

George Berkeley, an Anglo-Irish philosopher, proposed "immaterialism." His theory denies the existence of material substance. It suggests that the objects such as tables, chairs, space, and time are only ideas in the minds of perceivers, as a result cannot exist without being perceived. 
In the 18th century, the German philosopher Immanuel Kant developed a theory of knowledge. Kant came to the realization that space and time is not the objective feature of the world. Space and time is a framework used by us to organize our experience.

Under the influence of Austrian physicist and philosopher, Ernst Waldfried Josef Wenzel Mach, Einstein founded relativity theory. Einstein finds that space and time is relative. They are connected and can transform into each other. Space and time are two different aspects of one entity, spacetime.

Quantum physics challenges both Newton's and Einstein's concept about space and time in the most dramatic way. Quantum phenomena challenge Einstein's assumption that the transfer of information cannot exceed the speed of light. Quantum entanglement phenomena provide a way to transfer information instantly. Quantum entanglement means that the states of two or more vibrations are connected. When one or more vibrations are observed as certain states, the other vibrations with which it is quantum entangled will instantly turn into the state that is determined by the quantum entanglement no matter how far they are from each other.

Quantum physics also defies our normal ideas about space and time in a profound way. For instance, quantum phenomena reveal that we cannot measure time and energy simultaneously with the complete accuracy. We also cannot measure space and momentum simultaneously with the complete accuracy. This is the uncertainty principle in quantum physics.

The measurement of space and time is not trivial action in quantum physics. It is not simply assigning a number to a ruler, clock, or event. The observation and measurement of space and time is a creation process for the phenomena we observe.

\section{The Deeper Meaning and Function of Space and Time}

We suggest space and time is one of the fundamental yin-yang codes that manifest our universe from the emptiness. Time relates to the measurement of movement and change. It is the yang aspect of the yin-yang code. Space relates to the measurement of stillness and solidity. It is the yin aspect of the yin-yang code.

As we have mentioned before, quantum physics tells us that the world we observed is manifested by our measurement. Space and time relate to a pair of basic human duality actions. Fundamentally, time and space are two types of duality measurement. Time is the measurement of change. For instance, the measurement of the movement of sand in a container, the burning of incense, and the movement of sun and moon, has all been used as a measurement of time. The duration of a day is based on the measurement of rotation of the earth around itself. The length of lunar month is related to the movement of the moon around the earth. A year is the measurement of the evolution of earth around the sun. Space is the measurement of unchangeness and stillness. The length, height, and width of an object are the measurement of its unchangeness and stillness.

We suggest that space and time is a yin-yang pair. Space and time is opposite and relative. Change and stillness are opposite. They are relative because something may appear to change to you but to be still to others. Space and time is co-created because whenever we measure change we are referring to something unchanged. Whenever we measure unchanged, we are comparing to something changing. Therefore, space and time is inseparable and co-dependent. We propose that the interaction of space and time creates the universe. We will demonstrate this point below.

There is another yin yang pair also involved in the creation of our universe. This other yin yang pair is inclusion and exclusion. The inclusion and exclusion is a fundamental action we are doing all the time. We either include or exclude something in our actions. 
Let's examine our action more closely. We find that all measurement is based on these two basic yin yang pairs, space-time measurement, and inclusive-exclusive measurement. For instance, the measurement of velocity, acceleration, energy, momentum, temperature, spin, electricity, magnetic, mass, charge, force, and more are all variations of space and time measurement and inclusive and exclusive measurement. Some people may think the measurement of mass or temperature is unique. However, if you examine the different ways to measure mass or temperature, you will see that they are all different variations of the space and time measurement and inclusive and exclusive measurement. For instance, to measure mass with a balance, we put the matter to be measured on one side of the balance and the matter with known mass on the other side. When both sides of the balance are completely balanced, we obtain the completely equal mass on the both sides. In this way, the unknown mass is measured. In this measuring process, the space and time measurement is used to make sure that the two arms of the balance are equal and still. The inclusion and exclusion measurement is applied when one adds known mass to the balance.

In one word, all the measurements are different variation and combination of space and time measurement and inclusive and exclusive measurement. With the assumption that our action manifests the universe, these two yin yang pairs are the two fundamental yin yang pairs that manifest all of the phenomena in our universe.

\section{The Derivation of Wave Function of the Universe}

To see how the interaction of these two yin-yang pairs manifests our universe, we need to write down the action created by the interaction of these two yin yang pairs. In physics, action is an attribute of the dynamics of a physical system from which the equations of motion of the system can be derived. It depends on the trajectory, the path or history of the system.

The simplest action created by the interaction of the space-time yin yang pair is:

$$
\left.\mathrm{A}_{1}=\alpha\right\rfloor \Delta \tau \Delta \sigma
$$

Here we use the symbol $\sigma$ to represent space and the symbol $\tau$ to represent time. We use $\Delta \sigma$ and $\Delta \tau$ to represent the space and time duration to be measured. The symbol $\int$ represents the summation over space and time from the beginning $\tau=0$ and $\sigma=0$ till now $\tau=T$ and $\sigma=L$. Here $T$ is the age of our universe and $L$ is the horizon of our universe. And $\alpha$ is a constant. In our recent work, we have shown that:

$$
\alpha=1 /\left(1_{\mathrm{p}} \mathrm{t}_{\mathrm{p}}\right)
$$

Here $t_{p}$ is the Planck time. It is of the magnitude of $5.4 \times 10^{-44}$ second. It is the smallest time scale that naturally comes out of mathematic formula about nature so far. Here $1_{p}$ is the Planck length. It is of the magnitude of $1.6 \times 10^{-35}$ meter. It is the smallest length that naturally comes out of mathematics formula about nature so far.

To introduce the second yin yang pair into the action, it is important to realize that corresponding to the inclusion and exclusion yin yang pair, in nature there exist two types of particles, fermions, and bosons. Fermions have half (1/2) spin. They repel each other. They refuse to be in the same state. Particles such as electrons, muons, neutrinos, and quarks are fermions. Bosons have integer spin. They tend to clump. They love to stay at the same state together. Particles such as photons, gravitons, and gluons are bosons. Supersymmetry (SUSY) is the symmetry originally proposed to connect fermions and bosons. The normal time and space coordinates $\tau$ and $\sigma$ are of bosonic nature. If we assume each space or time coordinate has both the fermion (yang, repulsive) and boson (yin, clumping) parts, each time and space coordinate become two elements:

$$
\tau->\left(\tau, \theta_{\tau}\right)
$$




$$
\sigma->\left(\sigma, \theta_{\sigma}\right)
$$

Here we use $\theta_{\sigma}$ and $\theta_{\tau}$ to represent the fermion partner of space and time coordinate $\sigma$ and $\tau$. The $\theta_{\sigma}$ and $\theta_{\tau}$ can only take on the value 0 or 1 because they are repulsive and refuse to stay at the same place with another element. The $\sigma, \tau, \theta_{\sigma}$, and $\theta_{\tau}$ represent the four elements of the two yin yang pairs, space time yin yang pair and inclusion and exclusion yin yang pair.

The simplest action created by these two yin yang pairs is:

$$
\left.A_{2}=\alpha\right\rfloor \Delta \tau \Delta \sigma \Delta \theta_{\tau} \Delta \theta_{\sigma}
$$

The symbol $\int$ is to represent the summation over space $\sigma$ and time $\tau$ and $\theta_{\tau}$ and $\theta_{\sigma}$.

It is amazing to see that the action $A_{1}$ is similar to the action that creates string theory and the action $A_{2}$ is similar to the action that creates the superstring theory except for one difference. The action $A_{1}$ and $A_{2}$ sum over the life span and horizon of our universe while the string theory and superstring theory set the length of the string at the Planck scale $1_{\mathrm{p}}$ and they set the time to the infinity.

In Quantum physics, everything is mathematically represented by wave function. Using Feynman's path integral formulation, we can calculate the wave function $\Psi$ created by the action $A_{1}$ and $A_{2}$. The Feynman's path integral formulation of quantum physics is a way to calculate a quantum amplitude or wave function by summing over the all possible trajectories. It generalizes the action principle of classical mechanics and replaces the classical notion of a single definite trajectory with a summation over all possible trajectories. According to this method, the wave function created by the action $\mathrm{A}_{2}$ is in the form:

$$
\Psi=\sum_{\text {sum over all possible states }} C \exp \left(i \alpha A_{2}\right)
$$

Here $\mathrm{C}$ is a constant. The symbol $\sum$ is to sum over all possible paths and states. The equation (6) is the universal wave function formulation of superstring theory. In our previous work, we have shown that this difference and the universal wave function formulation of string theory enable us to derive the existence of dark matter and dark energy as well as estimate vacuum energy possibly consistent with the current experimental data.

According to superstring theory, also called M theory by people, all fundamental particles, interactions, and our observed space-time can be created from the combination of superstring action (5) and string action (6). Using the result obtained in string theory and $\mathrm{M}$ theory, it is not difficult to see that the universal wave function (6) also contains all the fundamental particles, interactions, and the observed space-time. In addition, the wave function tells a lot more about our universe than the current string theory. In our recent work, we have demonstrated that the wave function (6) tells us that our universe is a hologram. The observed space-time, particles, forces, and everything we observe is a projection from this hologram. From the universal wave function, we can show the existence of dark matter and dark energy in our universe. We can calculate the properties of dark matter, dark energy, and the cosmological constant. We find that our estimate of vacuum energy from equation (6) is consistent with the experimental data. The universal wave function also tells us that our observed space-time is made of different cycles. The large space and time cycles makes up the macroscopic phenomena such as planets, stars, galaxies, and large scale-structure in our universe. The small space and time cycles make up microscopic phenomena, such as quarks, electrons, photons, atoms, molecules and more. It also indicates and provides the mathematic description about the multiverse structure of our universe.

\section{Discussion}

In this work, we combine the ancient Chinese Tao wisdom about the creation with quantum physics. We 
propose and demonstrate mathematically that our universe is manifested from the emptiness through two basic human actions: measurement of space and time and measurement of inclusion and exclusion.

This work has great significance for the development of science. It provides a way to derive the grand unification theory, the one mathematic formula about our universe. It gives metaphysical understanding about string theory and makes it possible to bring out more testable results from string theory.

Philosophically, this work suggests and substantiates mathematically and scientifically that human action has manifested our universe from the emptiness. Our yin-yang action of space and time as well as inclusion and exclusion manifest our universe from Tao, the emptiness. Tao creates and includes all possibilities. Our action determines which possibility or possibilities are manifested from Tao.

This study expounds a deeper meaning and function of space and time. Space and time is a duality pair of the fundamental actions and codes that manifest our universe.

This work also provides insights to two important philosophical questions: Whether we can understand our world and why we can express the natural law mathematically. Mathematics is the human logic and derivation based on numbers and symbols. How can the universe follow human logic? The result of this study indicates that we can understand the universe because our own actions manifest the world we observe. Since mathematics is the universal language and logic of our actions and our mind, we can use mathematics to describe the universe and everything in our lives and the world. Tao is beyond our comprehension and description. But we can comprehend the universe and the world we experience because we create it.

More than 2500 years ago, Buddha reached the enlightenment when he gained the deep insight about how everyone and everything is created. Buddha realized that the physical reality is created from the emptiness through people's discrimination and attachment. Buddha's teaching has helped million people release their suffering and reach Nirvana. Our work demonstrates Buddha's profound wisdom and other similar spiritual wisdom about the creation mathematically and scientifically.

To understand scientifically and mathematically how we create the universe and the world we experience is one of the most empowering and enlightening knowledge. This understanding, wisdom, and knowledge can enlighten and liberate more people from the illusion and ignorance. It can relieve our suffering and bondage. It can uplift us to a higher level of existence. When we understand how we create our own reality, we will have the power to transcend our limitations and live a life with greater freedom and potential. We can manifest a better world with more love, peace, and harmony. We encourage more people to explore and contribute to the study of this subject and area.

\section{Works Cited}

Adler, Joseph A. Introduction to the Study of the Classic of Change (I-hsüehch'i-meng). Provo, Utah: Global Scholarly Publications, 2002. ISBN 1-59267-334-1.

Bennett, C., et al. "The Microwave Anisotropy Probe (MAP) Mission." Astrophysical Journal 583 (1): 1-23. arXiv: astro-ph/0301158. Bibcode 2003ApJ...583....1B. doi: 10.1086/345346 (2003a).

---. "First-Year Wilkinson Microwave Anisotropy Probe (WMAP) Observations: Foreground Emission." Astrophysical Journal Supplement 148 (1): 97-117. arXiv: astro-ph/0302208. Bibcode 2003ApJS..148...97B. doi: 10.1086/377252 (2003b).

Becker, Katrin, Becker, Melanie, and Schwarz, John. String Theory and M-Theory: A Modern Introduction. Cambridge University Press. ISBN 0-521-86069-5 (2007).

Dine, Michael. Supersymmetry and String Theory: Beyond the Standard Model. Cambridge University Press. ISBN 0-521-85841-0 (2007). 
Feynman, Richard Phillips, Hibbs, Albert R., and Styer, Daniel F. Quantum Mechanics and Path Integrals. Mineola, N.Y.: Dover Publications, 2010. 29-31. ISBN 0486477223.

Feynman, R. P. “The Space-Time Formulation of Nonrelativistic Quantum Mechanics.” Reviews of Modern Physics 20.2 (1948): 367-87.

Guth, Alan. "The Inflationary Universe.” Basic Books (1998).

Hinshaw, G., et al. "Three-Year Wilkinson Microwave Anisotropy Probe (WMAP1) Observations: Temperature Analysis." Astrophysical Journal Supplement 170 (2): 288-334. arXiv: astro-ph/0603451. Bibcode 2007ApJS..170..288H. doi: 10.1086/513698 (2007).

---. (WMAP Collaboration). "Five-Year Wilkinson Microwave Anisotropy Probe Observations: Data Processing, Sky Maps, and Basic Results." The Astrophysical Journal Supplement 180 (2): 225-45. arXiv: astro-ph/id=0803.0732. Bibcode 2009ApJS..180..225H. doi: 10.1088/0067-0049/180/2/225.<http://map.gsfc.nasa.gov/universe/uni_shape.html>. Feb. 2009.

Kaltenmark, Max. Lao Tzu and Taoism. Trans. Roger Greaves. Stanford: Stanford University Press. 1969.

Michael Green, John H. Schwarz, and Edward Witten. Superstring Theory. Cambridge University Press. Vol. 1: Introduction. ISBN 0-521-35752-7. Vol. 2: Loop Amplitudes, Anomalies and Phenomenology. ISBN 0-521-35753-5 (1987).

Polchinski, Joseph. String Theory. Cambridge University Press. Vol. 1: An Introduction to the Bosonic String. ISBN 0-521-63303-6. Vol. 2: Superstring Theory and Beyond. ISBN 0-521-63304-4 (1998).

Sha, Zhi Gang. Tao I: The Way of All Life. Heaven's Library/Atria, 2010.

---. Tao II: The Way of Healing, Rejuvenation, Longevity, and Immortality. Heaven's Library/Atria, 2010.

Sha, Zhi Gang and Xiu, Rulin. Soul Mind Body Science System. Benbella Books. ISBN 978-194036399-8 (2014).

---. "The Universal Wave Function Interpretation of String Theory." EPJ Web of Conferences.

---. "Tao Source Science.” EPJ Web of Conferences.

S. Perlmutter, G. Aldering, G. Goldhaber, R. A. Knop, P. Nugent, P. G. Castro, S. Deustua, S. Fabbro, A. Goobar, D. E. Groom, I. M. Hook, A. G. Kim, M. Y. Kim, J. C. Lee, N. J. Nunes, R. Pain, C. R. Pennypacker, R. Quimby, C. Lidman, R. S. Ellis, M. Irwin, R. G. McMahon, P. Ruiz-Lapuente, N. Walton, B. Schaefer, B. J. Boyle, A. V. Filippenko, T. Matheson, A. S. Fruchter, N. Panagia, H. J. M. Newberg, W. J. Couch (The Supernova Cosmology Project). "Measurements of Omega and Lambda from 42 High-Redshift Supernovae." The Astrophysical Journal 517 (2): 565-86. arXiv: astro-ph/9812133. Bibcode 1999ApJ...517..565P. doi: 10.1086/307221 (June 1999).

Unschuld, Paul U. Huang Di neijingsu wen: Nature, Knowledge, Imagery in an Ancient Chinese Medical Text. Berkeley and Los Angeles: University of California Press, 2003. ISBN 0-520-23322-0. 